\title{
Article \\ Characteristic Analysis, Simulation, and Experimental Comparison of Two Kinds of Circular Magnet Array in Energy Harvesting
}

\author{
Shengkai Guo (D), Shiqiao Gao*(D), Lei Jin *, Xueda Du (D), Qinghe Wu and Jie Shang
}

Citation: Guo, S.; Gao, S.; Jin, L.; Du, X.; Wu, Q.; Shang, J. Characteristic Analysis, Simulation, and Experimental Comparison of Two Kinds of Circular Magnet Array in Energy Harvesting. Appl. Sci. 2022, 12, 1962. https://doi.org/10.3390/ app12041962

Academic Editor: Gang Lei

Received: 8 January 2022

Accepted: 12 February 2022

Published: 13 February 2022

Publisher's Note: MDPI stays neutral with regard to jurisdictional claims in published maps and institutional affiliations.

Copyright: (c) 2022 by the authors. Licensee MDPI, Basel, Switzerland. This article is an open access article distributed under the terms and conditions of the Creative Commons Attribution (CC BY) license (https:// creativecommons.org/licenses/by/ $4.0 /)$.

\author{
State Key Laboratory of Explosion Science and Technology, Beijing Institute of Technology, Beijing 100081, China; \\ guoshengkai9@163.com (S.G.); a929632027@outlook.com (X.D.); 3120160078@bit.edu.cn (Q.W.); \\ 13664316055@163.com (J.S.) \\ * Correspondence: gaoshq@bit.edu.cn (S.G.); jinlei@bit.edu.cn (L.J.)
}

\begin{abstract}
The circular Halbach array is of great application value in electromagnetic energy conversion. The Halbach array can be formed with multiple magnets according to a specific method. The magnetic flux intensity of the magnet array is concentrated and enhanced in a certain area. The concentrated magnetic flux intensity could lead to a higher rate of change in the magnetic flux when the magnet and the coil move relatively. The spatial arrangement, structural dimensions, and residual flux density of the Halbach array are the main factors affecting its performance. Some areas with almost no magnetic induction in the Halbach array affect the performance of electromagnetic energy conversion. This paper conducted a comparative study of the Halbach array and the two-directional magnet array in terms of energy conversion. The root mean square (RMS) value and phase of the induced voltage in the coil were analyzed by a finite element method. Simulation shows that the RMS value of the induced voltage in the coil is equal when the gap is $1.55 \mathrm{~mm}$ and the REMEH adopts either of the two magnet arrays. The two-directional array has advantages in the energy harvesting performance when the gap is less than $1.55 \mathrm{~mm}$, while the Halbach array has advantages in the energy harvesting performance when the gap is greater than $1.55 \mathrm{~mm}$. The experimental verification of the designed prototype was implemented in this study. The experimental voltage is in good agreement with the simulation voltage. The threshold value of the gap in the experiment is about $2 \mathrm{~mm}$, which is slightly higher than the simulation value. This characteristic has a certain reference value for the application of the Halbach array in the electromagnetic field.
\end{abstract}

Keywords: Halbach array; two directional magnet array; magnetic flux density comparison; voltage comparison

\section{Introduction}

Electromagnetic energy harvesters generate induced voltage according to Faraday's Law [1-5]. The arrangement of the magnet and the relative movement speed between the magnet and the coil are the direct influencing factors in electromagnetic energy harvesters. The Halbach array is widely used in the field of electromagnetic energy conversion, which has been a hot research topic in recent years. The Halbach array has the characteristic that the magnetic flux density increases on one side and decreases on the other side because it uses a certain regular arrangement of magnets. On the side where the magnetic induction intensity increases, the maximum magnetic induction intensity is improved, and the distribution range is expanded. On the other side, the magnetic induction intensity is reduced and the distribution range is reduced.

Researchers have proposed a variety of Halbach arrays to be used in electromagnetic energy conversion. Salauddin [6-10] proposed a hybrid energy harvester that includes a stacked in-plane Halbach array. In addition, some in-plane Halbach arrays [11-13] and cylindrical Halbach arrays [14-18] have been presented and utilized. Qiu [19] and 
Liu $[20,21]$ designed an electromagnetic energy harvester employing a Halbach array and a coil. The coil, which is attached to the end of the cantilever beam, vibrates and results in the generation of induced voltage. Zhu $[22,23]$ proposed an electromagnetic energy harvesting structure that includes a meander spring and an in-plane Halbach array.

Some researchers have studied the application of Halbach magnet arrays arranged in a circle in a rotating environment. Jae [24] reported a non-resonant finger-triggered energy harvester with a disc-shaped Halbach array. Wang [25] proposed a circular Halbach array magnetic disk, which was used in a natural-frequency self-tuning energy harvester. Zhang [26,27] proposed a circular Halbach array for monitoring the health condition of bearings. Because of the low fabrication cost, 16 cubic magnets instead of arc-shaped magnets were embedded in a ring area to form a Halbach array. The maximum magnetic induction intensity is increased and the minimum magnetic induction intensity is decreased on one side of the Halbach array ring. The induced voltage of each of two coils in the three coils exhibits a phase difference of 120 degrees. The induced voltage of the three coils forms a three-phase alternating current, which can be a delta connection or a star connection according to the application requirements. Maamer [28] proposed a circular Halbach array to concentrate the magnetic flux density in the radial direction. The coils are arranged inside of the circular Halbach array in order to better integrate the system. The studies above show that the use of a circular Halbach array in a rotational electromagnetic energy harvester (REMEH) can improve the electromagnetic energy harvesting performance to a certain extent.

However, there are also some areas with almost no magnetic induction in the abovementioned Halbach arrays even though the magnetic induction of the magnetic poles was concentrated. In fact, the magnetic flux density of a circular Halbach array is affected by many factors. The size, shape, material, residual magnetic flux density, and gap between the cube magnets are factors that affect the magnetic flux density of the Halbach array. The magnetic induction intensity at the magnetic poles is close to the residual magnetism of the NdFeB magnet. In addition, reducing the gap between the magnet and the coil is a common way to improve the electromagnetic induction. The above-mentioned studies utilized the excellent effects of the circular Halbach array. However, there is little research comparing the effects of the magnet arrangement on energy conversion. The abovementioned research did not reveal the advantages of a Halbach array composed of cubic magnet blocks. The advantages and disadvantages of the circular Halbach array have not been well established, which would be helpful for expanding the application of the Halbach array. The contributions of this work are as follows: (1) a theoretical model of electromagnetic energy harvesting in cylindrical coordinates is analyzed and established based on the coordinates of cubic magnet nodes; (2) the magnetic flux density and energy harvesting performance of two magnet arrays in a rotating environment are compared in a simulation and an experiment; and (3) the phase of the induced voltage in the coils is analyzed when the two magnet arrays are applied in energy acquisition.

This paper compares the Halbach array with the two-directional magnet array in terms of various aspects of energy conversion. The magnetic flux density, distribution range, and induced voltage under different parameters of the two magnet arrays are compared. It is concluded that the Halbach array adopted in this paper has advantages in energy harvesting only when the distance between the magnet and the coil is sufficiently large. The design and corresponding analysis are presented in Section 2. Section 3 presents a comparison of simulations in COMSOL Multiphysics between the two magnet arrays, including the magnetic flux density and voltage response under different gaps and rotating speeds. Experiments are described and verified in Section 4. Finally, a conclusion is drawn in Section 5. 


\section{Electromagnetic Energy Harvester: Principles and Design}

\subsection{Structural Design}

A three-dimensional structure is proposed in Figure 1. Sixteen magnets were arranged in two ways to form two magnet array rings (a Halbach array and a two-directional array). Four cubic magnets were arranged in each group, and the Halbach array was composed of four groups. The magnetization directions of the four magnets in each group were specific. The magnetization direction of eight magnets in the two-directional array is the $Z$ axis, and the magnetization direction of the other eight magnets is opposite to the $Z$ axis. The coil face of the magnet array was fixed on the stator. The magnet array is the rotating part. The stator and the rotor had the same axis. A change in the magnetic flux in the coil results in the generation of induced voltage when the magnet array rotates.

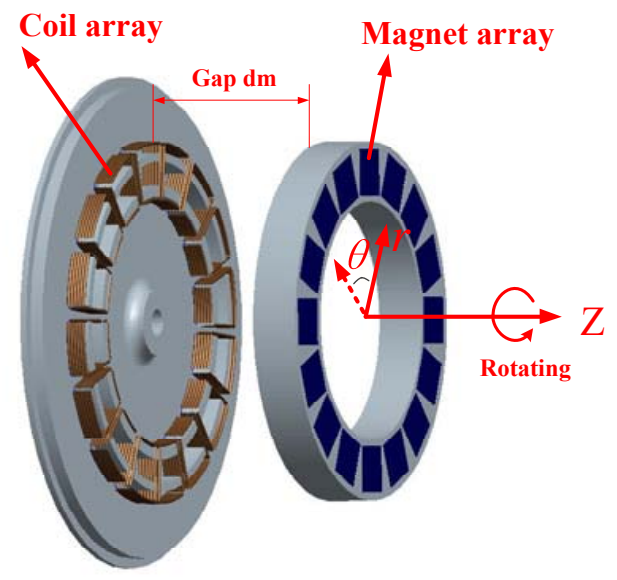

(a)

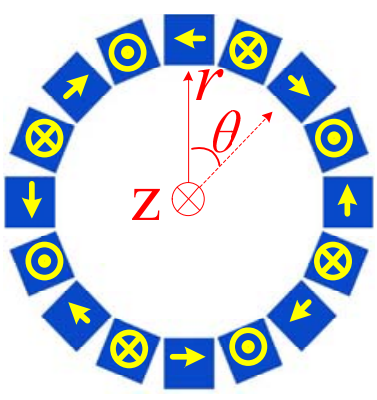

(b)

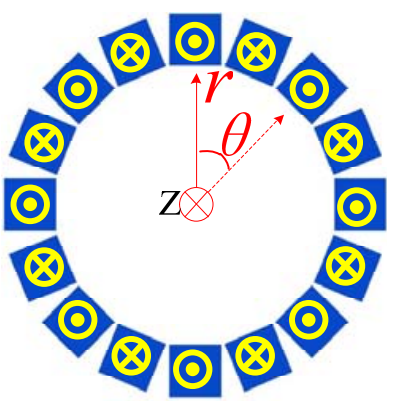

(c)

Figure 1. Schematic of the proposed REMEH with coils and magnets. (a) Electromagnetic energy harvesting structure; (b) Halbach array; (c) two-directional magnet array.

\subsection{Modeling of the Energy Harvester}

In this section, the analysis of the electromagnetic energy harvester and the calculation of the magnetic flux density are described. The magnetic nodes theory in [27-29] indicates that a magnetic field can be calculated by eight nodes of coordinates. The spatial component of the magnetic field at point $P(r, \theta, z)$ is:

$$
B_{z}(r, \theta, z)=\sum_{k=1}^{8} \frac{\varepsilon_{k}}{\pi} \arctan \left(\frac{\delta_{k} \sigma_{k}}{d d_{z}}\right)
$$

where $\delta_{k}$, one spatial component of the distance in cartesian coordinates between point $\mathrm{P}$ and the node can be expressed as $r \cos \theta-r_{k} \cos \theta_{k}, \sigma_{k}$, another spatial component of the distance in cartesian coordinates between point $P$ and the node can be expressed as $r \sin \theta-r_{k} \sin \theta_{k}, d_{z}$, the third spatial component of the distance between point $P$ and the node can be expressed as $z-z_{k}, d$, the spatial distance between point $P$ and the 
node, can be expressed as $\sqrt{\left(r \cos \theta-r_{k} \cos \theta_{k}\right)^{2}+\left(r \sin \theta-r_{k} \sin \theta_{k}\right)^{2}+\left(z-z_{k}\right)^{2}}$, and $\varepsilon_{k}$ is the magnetization of each node. The values of the magnetization of adjacent nodes are the opposite.

A circular magnet rotates around the $Z$ axis. The magnetic flux in the $Z$ direction of a turn of the coil is:

$$
\phi(t)=\int_{r_{1}}^{r_{2}} \int_{0}^{\pi / 8} B_{z}(r, \beta(t)+\alpha, z) r d \alpha d r
$$

where $r_{1}$ and $r_{2}$ are the inner radius and the outer radius of the circular magnet array, respectively, the gap between the magnet array and the coils is described as $d_{m}, z_{h}$ is the width of the coils, and $\beta(t)$ is the angular displacement between coil $i$ and the magnet as shown in Figure 2. The induced voltage in coil $i$ is caused by Faraday's law [30] and can be indicated as:

$$
\begin{aligned}
& U_{i}(t)=\int_{d_{m}}^{d_{m}+z_{h}}-\frac{d \phi(t)}{d t} n_{z} d z=\int_{d_{m}}^{d_{m}+z_{h}}-\frac{d \phi(t)}{d \beta(t)} \frac{d \beta(t)}{d t} n_{z} d z \\
& =-\omega \int_{d_{m}}^{d_{m}+z_{h}} \int_{r_{1}}^{r_{2}} \int_{0}^{\pi / 8} n_{z} \frac{\partial B_{z}(r, \beta(t)+\alpha, z)}{\partial \beta} r d \alpha d r d z \\
& =-n_{z} \omega \int_{d_{m}}^{d_{m}+z_{h}} \int_{r_{1}}^{r_{2}} B_{z}(r, \beta(t)+\alpha, z) \mid \begin{array}{c}
\pi / 8 \\
0
\end{array} \quad r d r d z \\
& =-n_{z} \omega \int_{d_{m}}^{d_{m}+z_{h}} \int_{r_{1}}^{r_{2}} \Delta B_{z} r d r d z
\end{aligned}
$$

where $n_{z}$ is the number of coil turns per unit of height. It can be seen from formula (3) that the energy conversion efficiency can be judged by $\Delta \mathrm{B}$ at the coil when the coil density, rotation speed, and radius parameters are all fixed for a certain rotating electromagnetic energy harvester.

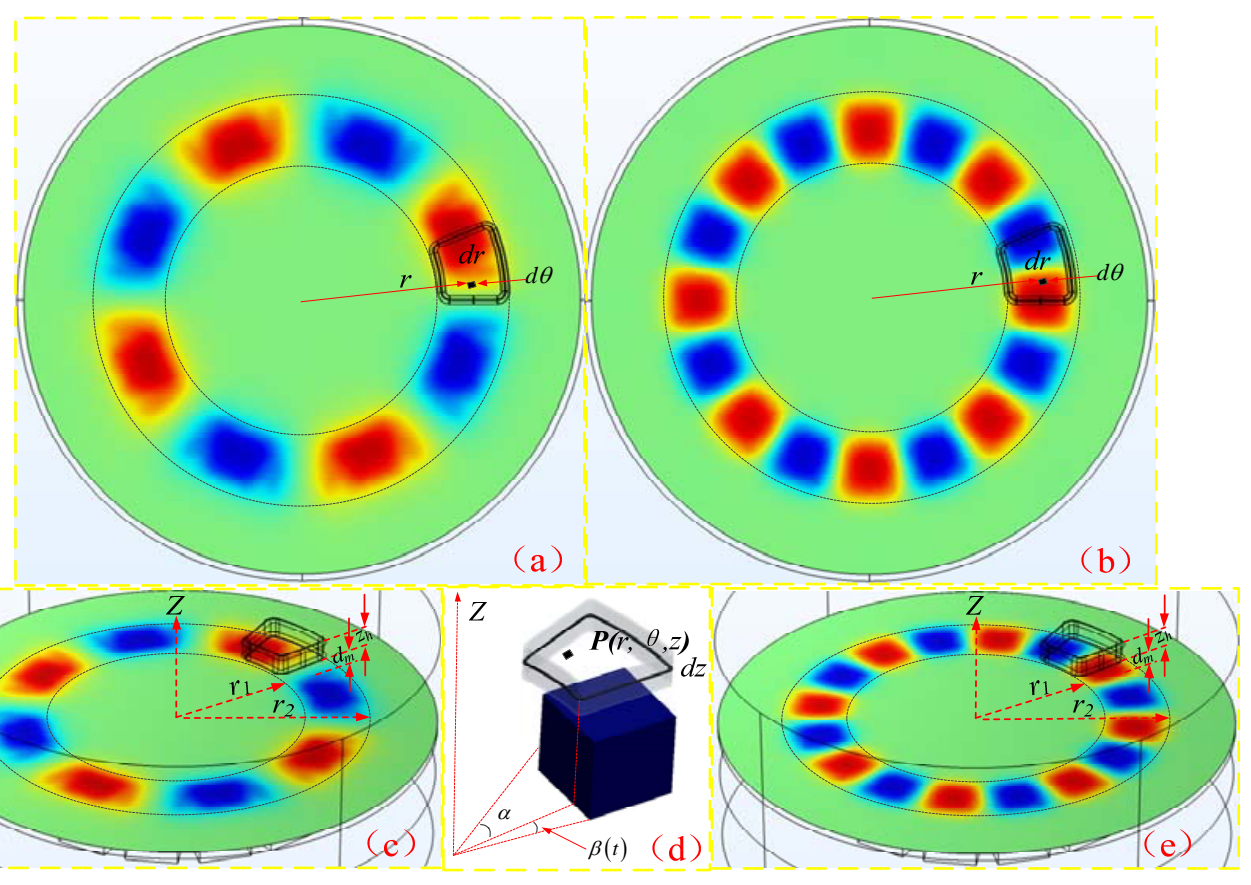

Figure 2. Dynamic state of an energy harvester with multiple parameters: (a) top view of the magnets and coil with the Halbach array; (b) top view of the two-directional magnet array and coil; (c) threedimensional view of the magnets and coil with the Halbach array in a dynamic response state; (d) three-dimensional view of cylindrical coordinates; (e) three-dimensional view of the magnets and coil with the two-directional array in a dynamic response state. 


\section{Analysis of the Electromagnetic Energy Harvester}

\subsection{Magnetic Flux Simulation Comparison}

Figure 3a-d present the magnetic flux density of the Halbach array at four different gaps. The maximum magnetic flux density decreases as the gap increases. The maximum magnetic flux density at a gap of $1 \mathrm{~mm}, 2 \mathrm{~mm}, 3 \mathrm{~mm}$, and $4 \mathrm{~mm}$ is $0.48277 \mathrm{~T}, 0.36561 \mathrm{~T}$, $0.27524 \mathrm{~T}$, and $0.20551 \mathrm{~T}$, respectively.

Figure $3 \mathrm{e}-\mathrm{h}$ demonstrate the magnetic flux density of the two-directional magnet array at four different gaps. The maximum magnetic flux density at a gap of $1 \mathrm{~mm}, 2 \mathrm{~mm}, 3 \mathrm{~mm}$, and $4 \mathrm{~mm}$ is $0.40641 \mathrm{~T}, 0.27688 \mathrm{~T}, 0.18382 \mathrm{~T}$, and $0.12046 \mathrm{~T}$, respectively.

A comparison of the magnetic flux density under different gaps between the Halbach array and the two-directional array is shown in Table 1. As the gap diameter increases, the magnetic flux density of the Halbach array decreases more slowly than that of the two-directional magnet array. The maximum magnetic flux density of the Halbach array is almost 2 times the maximum magnetic flux density of the two-directional array when the gap diameter is $5 \mathrm{~mm}$. The difference in magnetic flux density at the magnetic poles of the two magnet arrays decreases as the gap diameter decreases. The maximum magnetic flux density of the two-directional array is close to the maximum magnetic flux density of the Halbach array when the gap diameter reaches $1 \mathrm{~mm}$. Figure $4 \mathrm{a}, \mathrm{b}$ show that the magnetic flux density at the poles of the two-directional array gradually approaches the magnetic flux density at the poles of the Halbach array when the gap diameter approaches 0 . The magnetic flux density at the poles of the Halbach array is higher than that of the two-directional array at the same gap diameter. Figure $4 \mathrm{c}$,d show the magnetic flux density versus the gap diameter at the poles of the two magnet arrays.

Table 1. Comparison of the peak magnetic flux density between the Halbach array and the twodirectional array at different gap diameters.

\begin{tabular}{|c|c|c|c|c|c|}
\hline Gap Diameter $\quad$ Bz (T) & $1 \mathrm{~mm}$ & $2 \mathrm{~mm}$ & $3 \mathrm{~mm}$ & $4 \mathrm{~mm}$ & $5 \mathrm{~mm}$ \\
\hline Halbach array & 0.48277 & 0.36561 & 0.27524 & 0.20551 & 0.15478 \\
\hline Two-directional array & 0.40641 & 0.27688 & 0.18382 & 0.12046 & 0.07971 \\
\hline
\end{tabular}

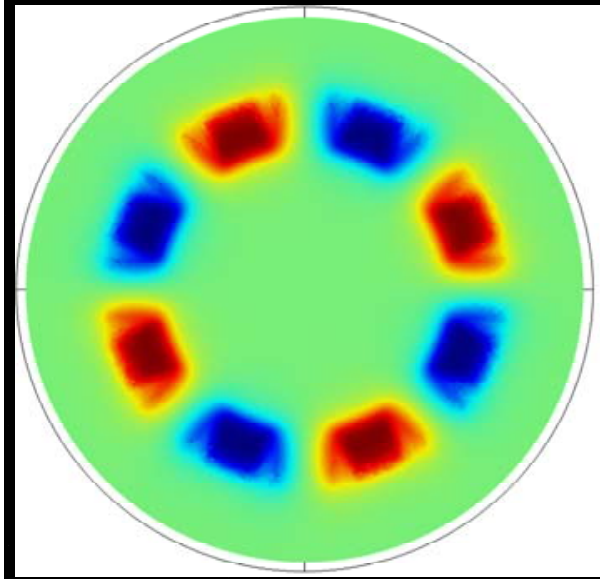

(a) $\mathrm{d}=1 \mathrm{~mm}$
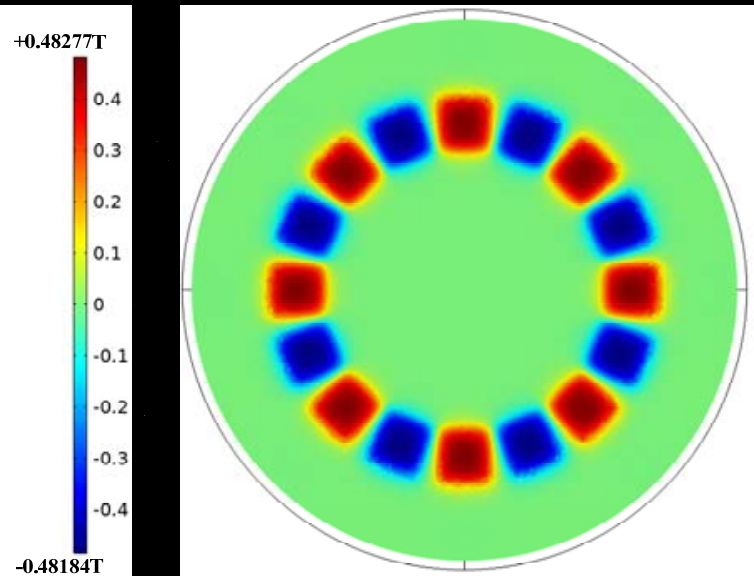

(e) $\mathrm{d}=1 \mathrm{~mm}$

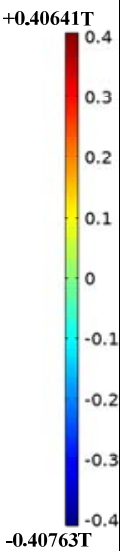

$-0.40763 \mathrm{~T}$

Figure 3. Cont. 


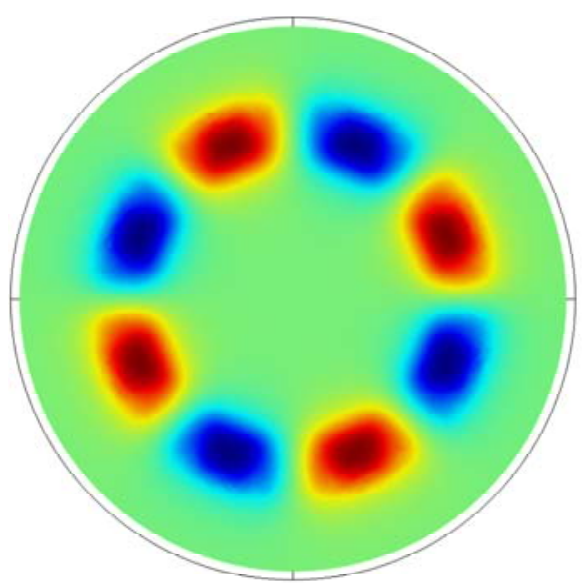

(b) $\mathrm{d}=2 \mathrm{~mm}$

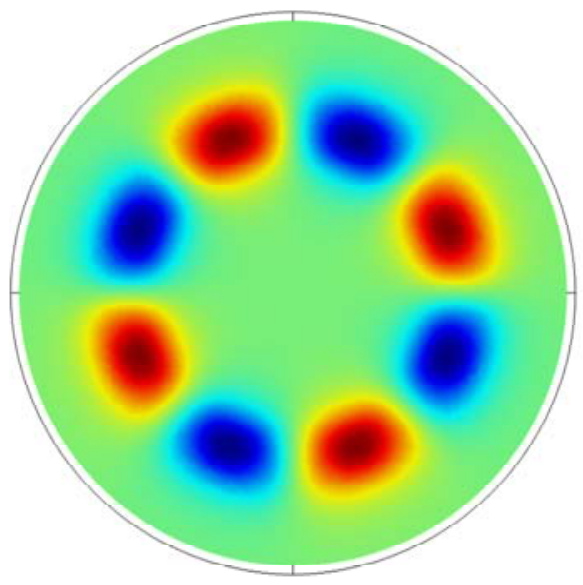

(c) $\mathrm{d}=3 \mathrm{~mm}$

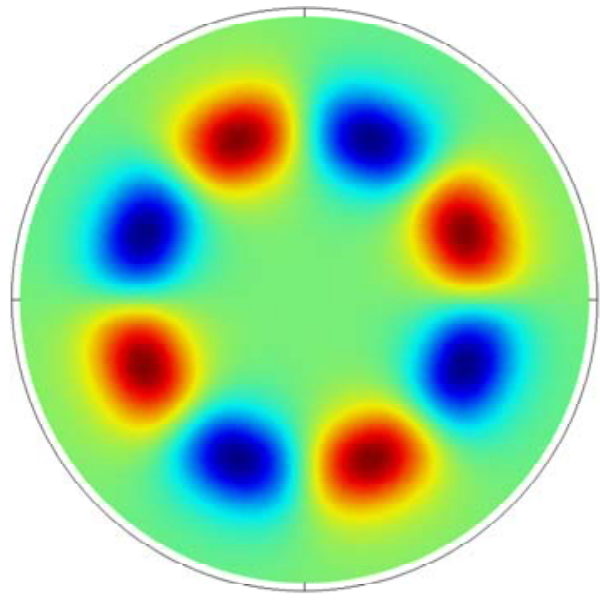

(d) $\mathrm{d}=4 \mathrm{~mm}$
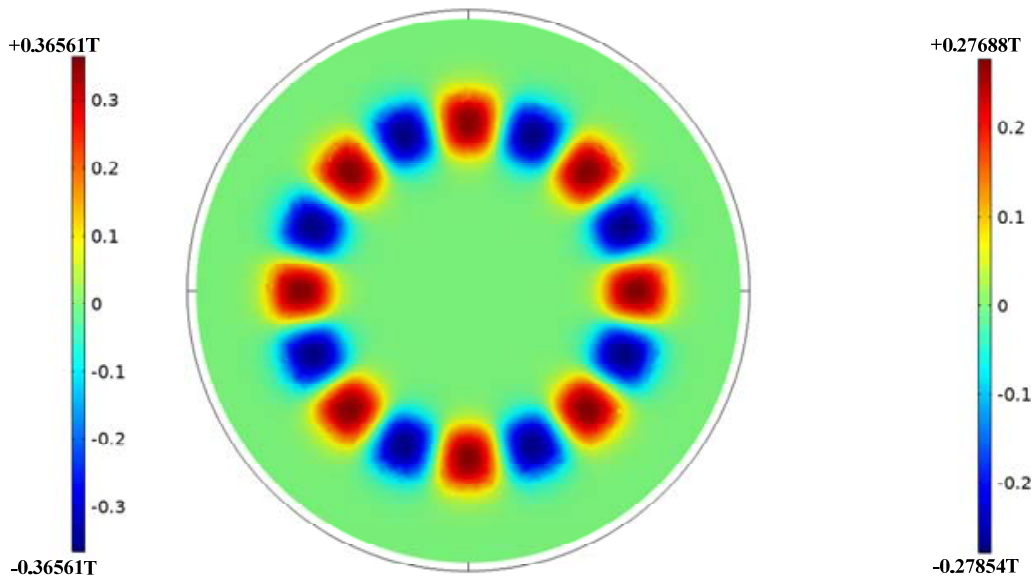

(f) $\mathrm{d}=2 \mathrm{~mm}$
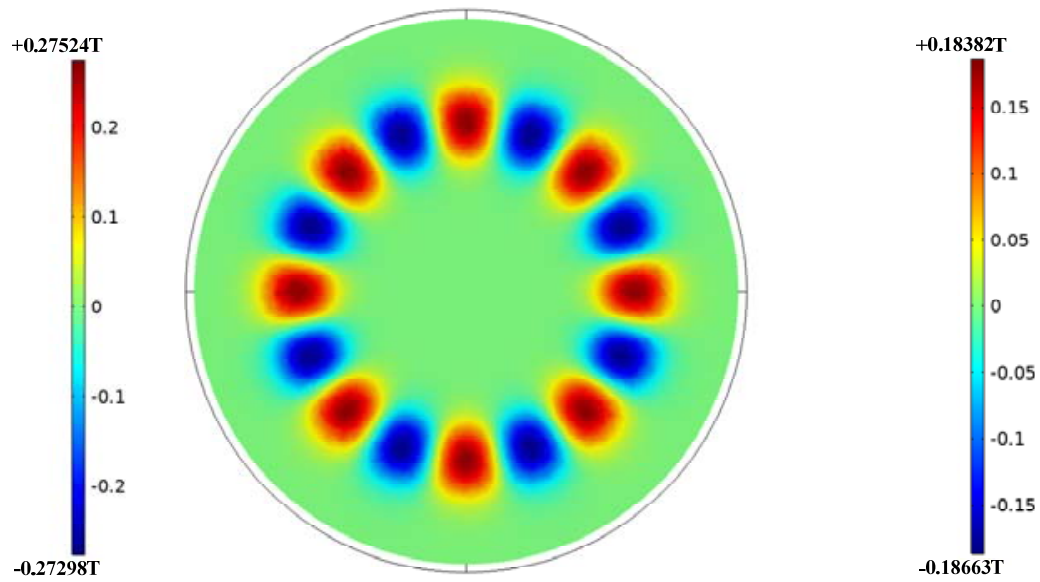

(g) $\mathrm{d}=3 \mathrm{~mm}$
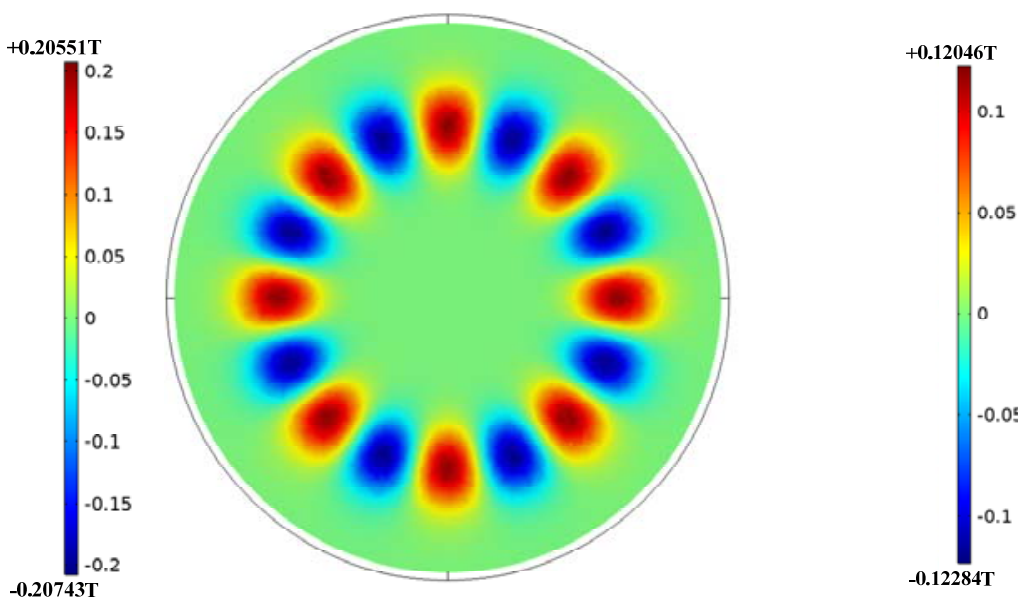

(h) $\mathrm{d}=4 \mathrm{~mm}$

Figure 3. Comparisons of the magnetic flux density under different gaps between the two magnet arrangements: (a-d) magnetic flux density of the Halbach array; and (e-h) magnetic flux density of the two-directional magnet array. 


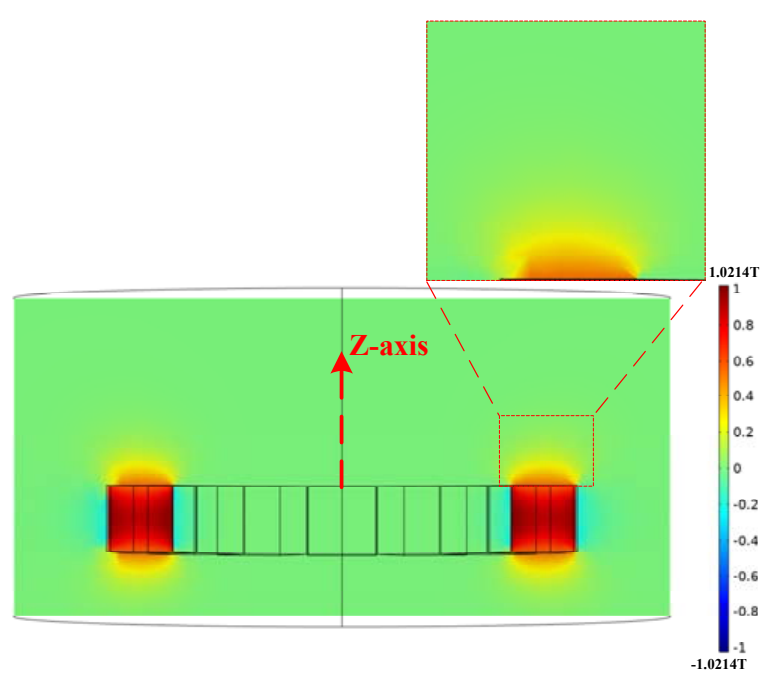

(a)

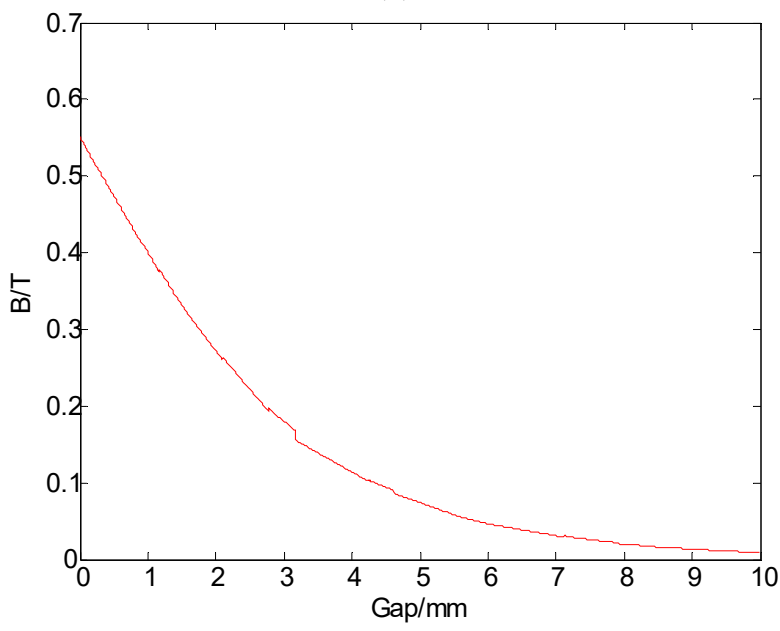

(c)

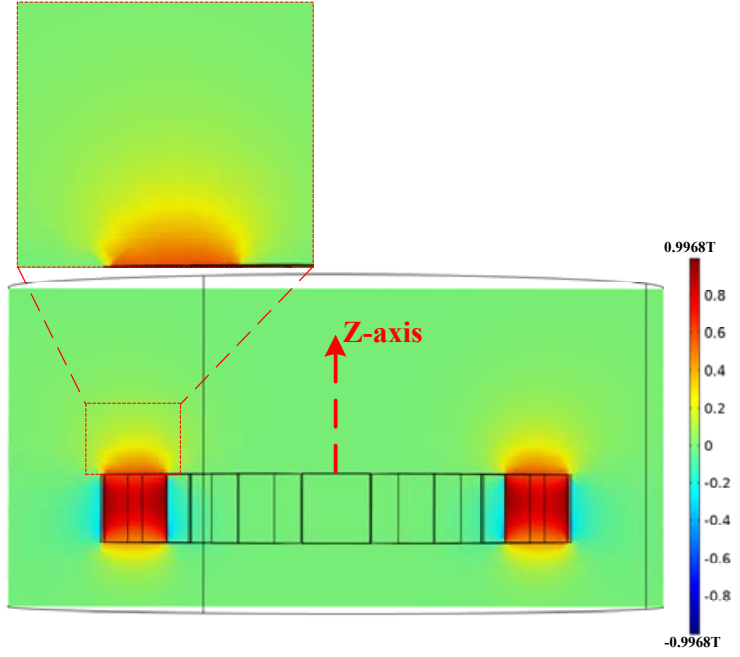

(b)

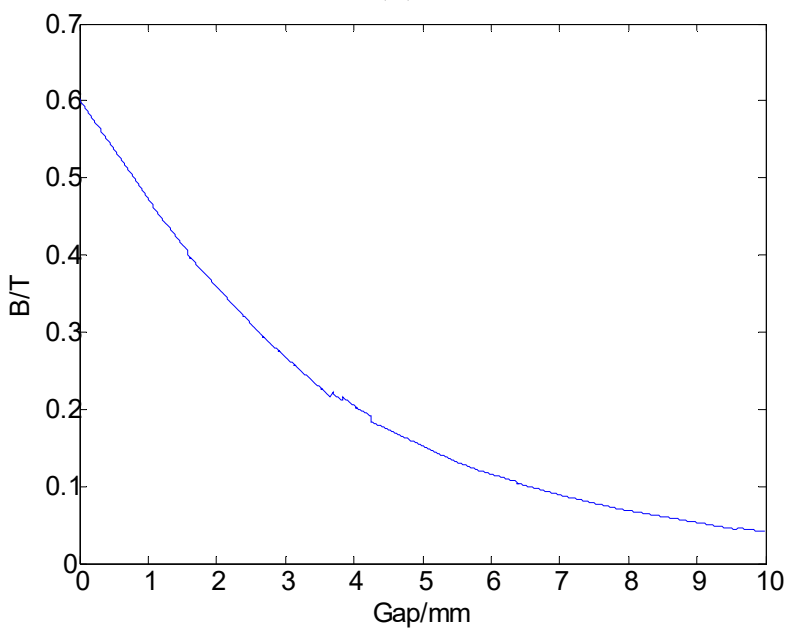

(d)

Figure 4. Sectional view of the magnetic flux density at the two magnet arrays' poles: (a) twodirectional magnet array; (b) Halbach array; (c) magnetic flux density at the poles of the twodirectional magnet array; (d) magnetic flux density at the poles of the Halbach array.

Figure 5 indicates the magnetic flux density of Halbach array versus the angle at different gaps when the radius is $21.5 \mathrm{~mm}$. Figure 6 indicates the magnetic flux density of two directional magnet array versus the angle at different gaps when the radius is $21.5 \mathrm{~mm}$.

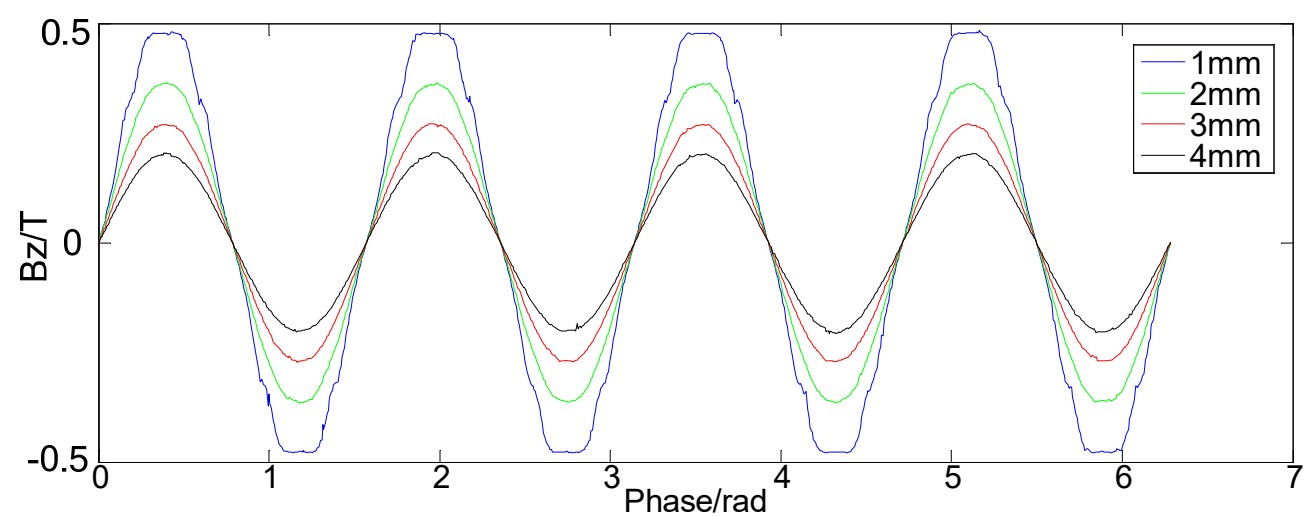

Figure 5. Magnetic flux density versus angle at four different gaps for the Halbach array. 


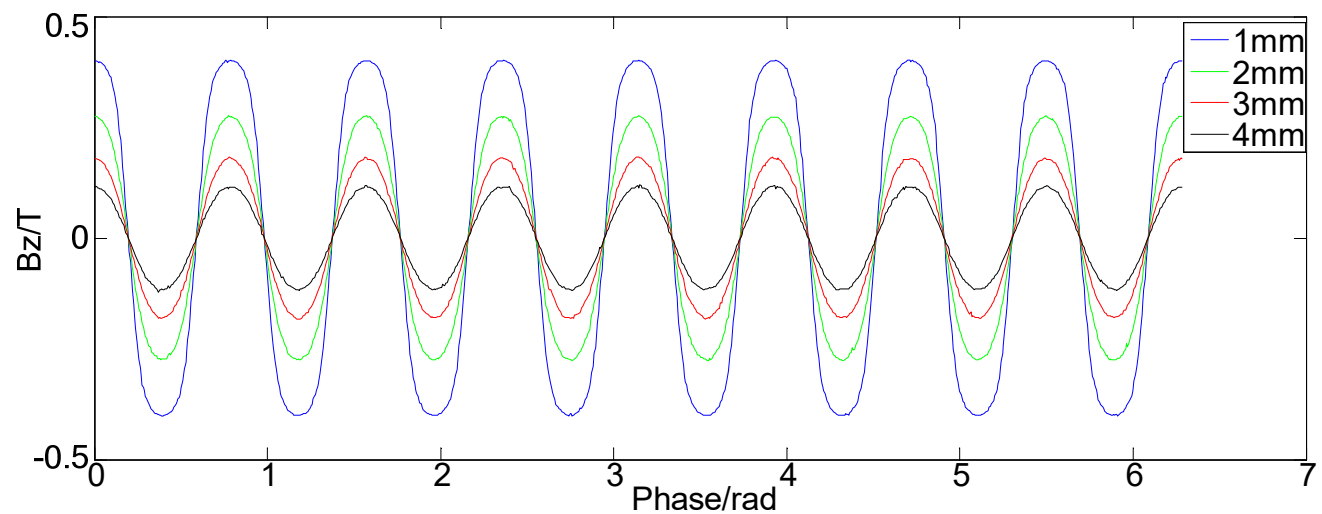

Figure 6. Magnetic flux density versus angle at four different gaps for the two-directional magnet array.

$\Delta \mathrm{B}$, the difference in magnetic flux density, was calculated from the magnetic flux density at the junction of the coil and the selected arc, which had a radius of $21.5 \mathrm{~mm}$. The calculated $\Delta \mathrm{B}$ also changed periodically with the rotation of the coil. As Figure 7 shows, the peak value of the calculated $\Delta \mathrm{B}$ by the two-directional array is larger than that of the Halbach array when the gap diameter is $1 \mathrm{~mm}$. The peak value of the calculated $\Delta \mathrm{B}$ by the Halbach array is larger than that of the two-directional array when gap diameter is $3 \mathrm{~mm}$. The magnetic flux difference $\Delta \mathrm{B}$ reflects the change in magnetic flux and also affects the induced voltage directly.

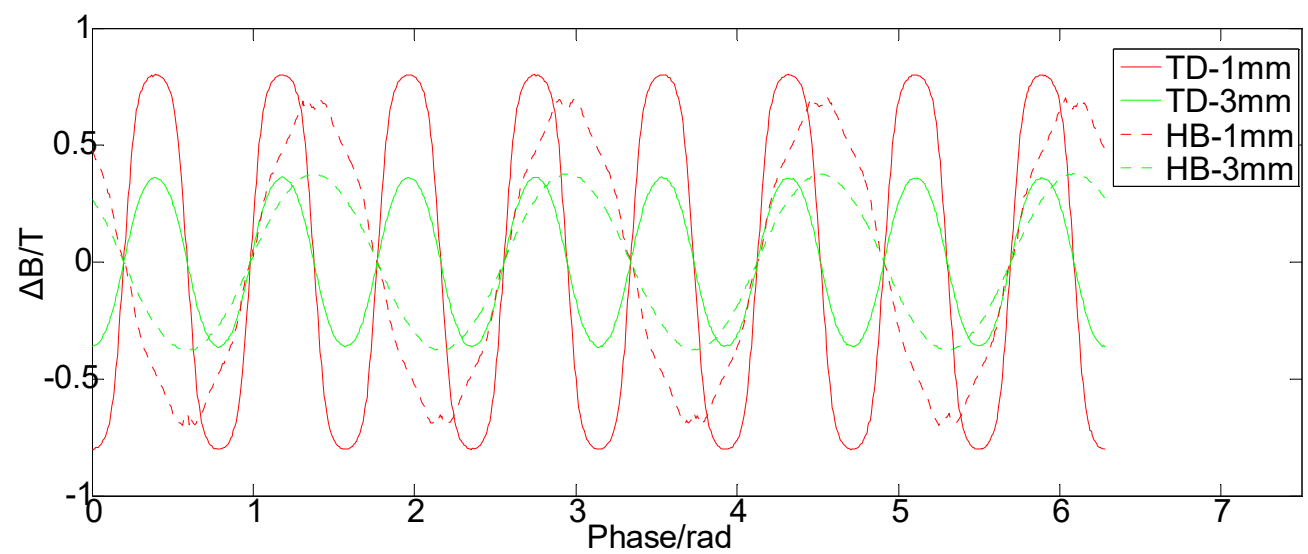

Figure 7. The relationship between the $\Delta \mathrm{B}$ value and the angle when the two arrays are applied with different gap diameters. TD $-1 \mathrm{~mm}$, two directional array with a gap diameter of $1 \mathrm{~mm} ; \mathrm{HB}-1 \mathrm{~mm}$, Halbach array with a gap diameter of $1 \mathrm{~mm}$; TD-1 mm, two-directional array with a gap diameter of $3 \mathrm{~mm}$; HB-1 mm, Halbach array with a gap diameter of $3 \mathrm{~mm}$.

\subsection{Voltage Response Analysis}

In this section, we analyze and compare the induced voltage in a single coil under multiple parameters. The phase difference in the coil's induced voltage at different positions is analyzed.

The periodic curve of induced voltage versus time in the coil of the REMEH with the Halbach array is shown in Figure 8. When the gap between the coil and the magnet array increases, the amplitude of the induced voltage decreases, while the frequency and period of the induced voltage remain unchanged. The decrease in magnetic induction as the gap increases is the reason for the decrease in the induced voltage in the coil. 


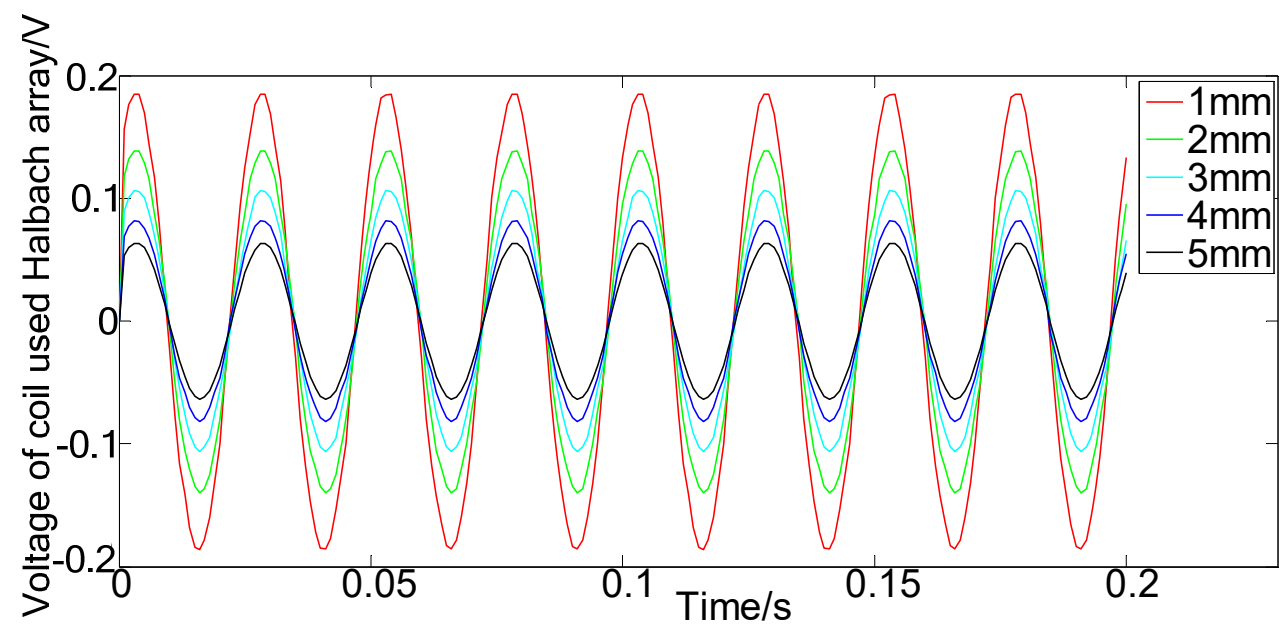

Figure 8. Induced voltage in the coil of the REMEH with the Halbach array versus time under different gaps at a rotation speed of $10 \mathrm{r} / \mathrm{s}$.

The periodic curve of induced voltage versus time in the coil of the REMEH with the two-directional magnet array is shown in Figure 9. The magnetization of eight magnets in the two-directional magnet array is in the z-axis direction, and the magnetization of the other eight magnets is in the opposite direction. The period number of the induced voltage in the coil with the two-directional magnet array is twice that of the coil with the Halbach array. The amplitude of the induced voltage in the coil using the Halbach array exceeds the induced voltage in the coil using the two-directional magnet array when the gap diameter is $2 \mathrm{~mm}$.

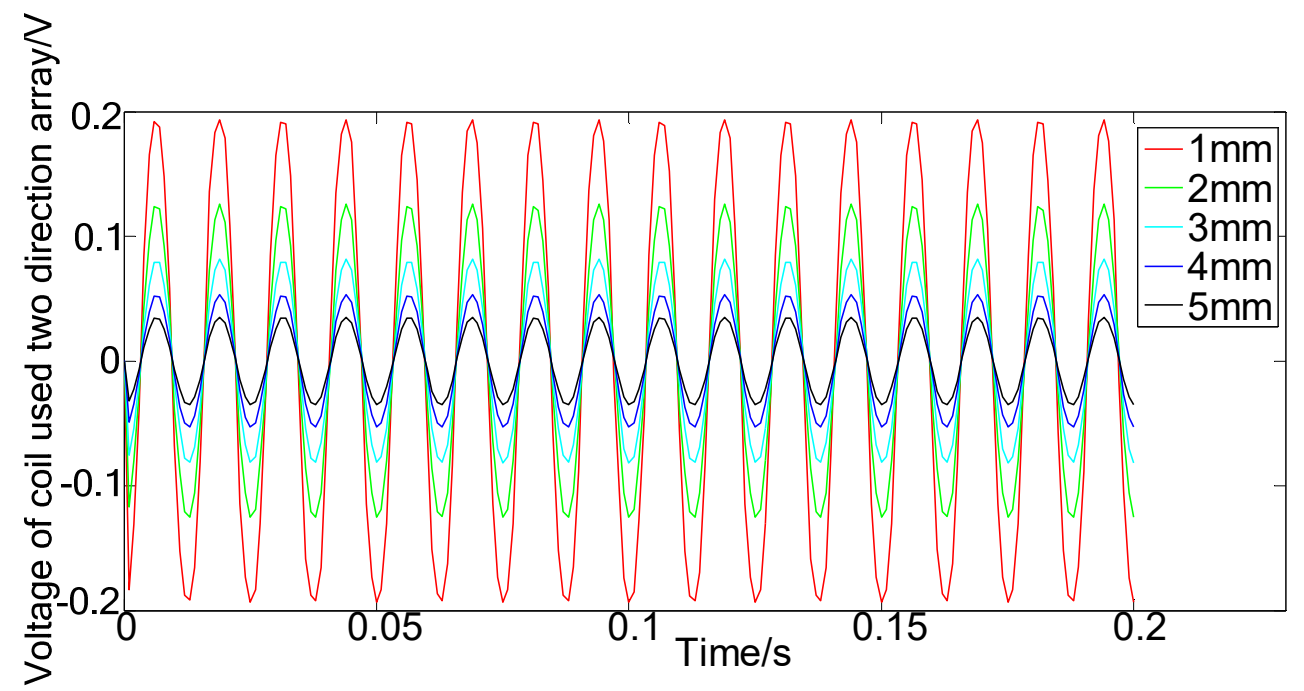

Figure 9. Induced voltage in the coil of the REMEH with the two-directional magnet array under different gaps at a rotation speed of $10 \mathrm{r} / \mathrm{s}$.

Different rotation speeds result in different amplitudes and frequencies of the periodic induced voltage under the same gap diameter. The induced voltage's amplitude doubles and the frequency doubles when the speed is increased from $5 \mathrm{r} / \mathrm{s}$ to $10 \mathrm{r} / \mathrm{s}$, as is shown in Figures 10 and 11.

The induced voltage in the coil of the REMEH using the Halbach array exhibits four different phases under the same gap diameter and rotation speed, as shown in Figures 12 and 13. The different initial positions of the coil caused four different voltage phases. The amplitude, frequency, and RMS value of the voltage in each coil are the same. 


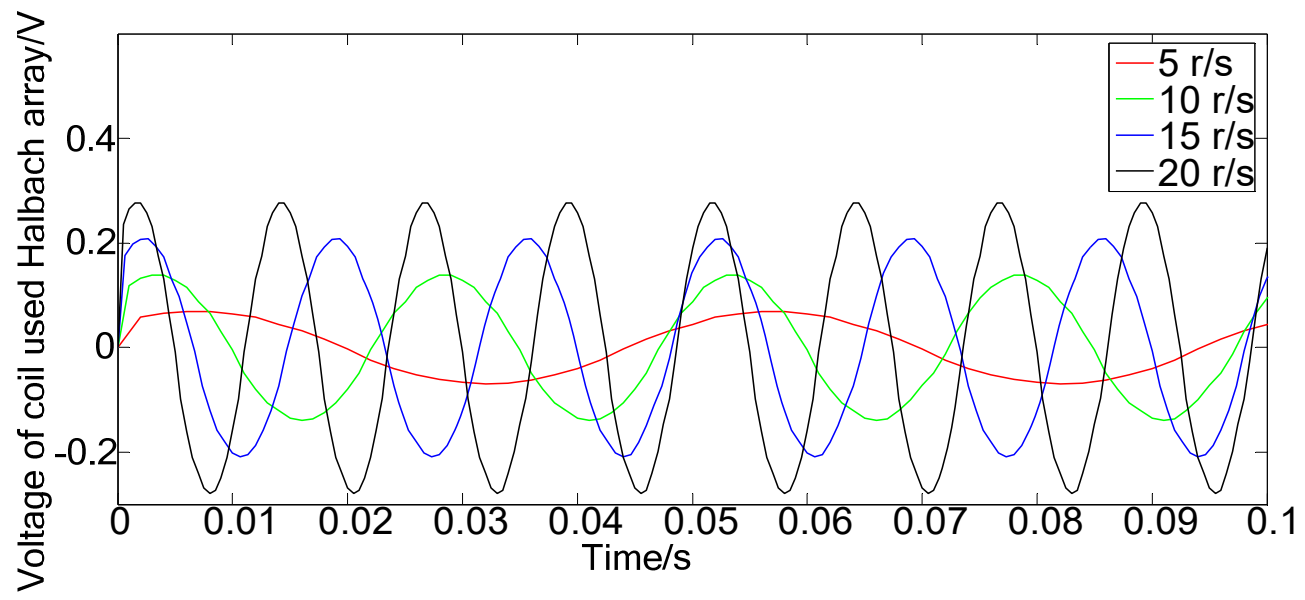

Figure 10. Induced voltage in the coil of the REMEH with the Halbach array versus time under different rotation speeds when the gap is $2 \mathrm{~mm}$.

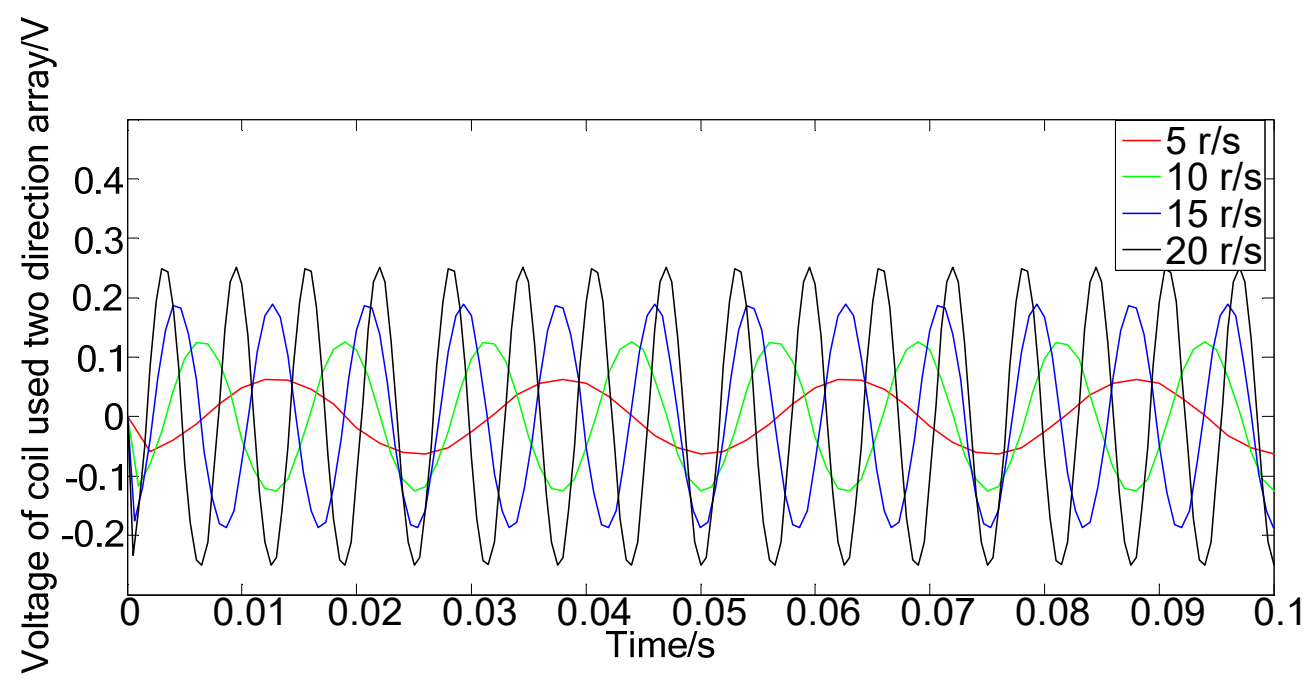

Figure 11. Induced voltage in the coil of the REMEH with the two-directional magnet array versus time under different rotation speeds when the gap diameter is $2 \mathrm{~mm}$.

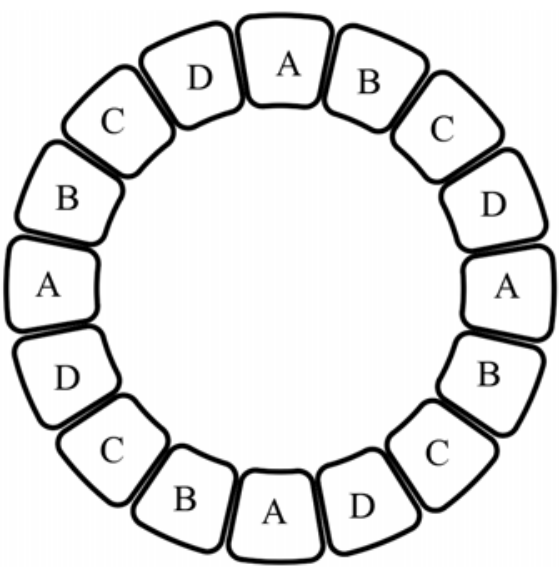

(a)

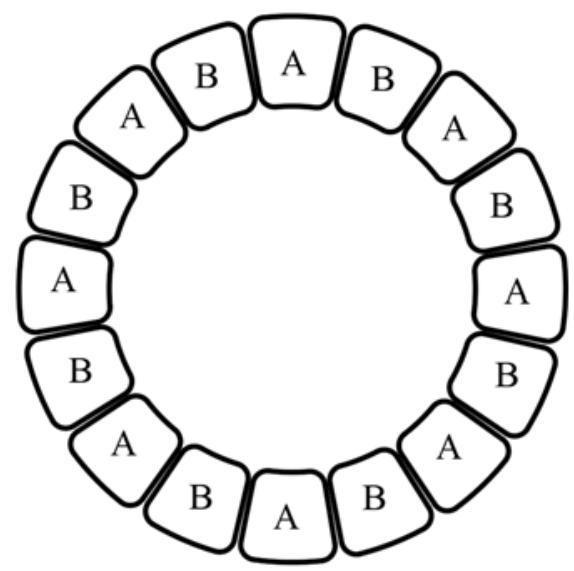

(b)

Figure 12. Four phases of the induced voltage in the coil of the REMEH with the Halbach array and two phases of the induced voltage in the coil of the REMEH with the two-directional array. The number of turns of each coil in the calculation is 50. (a) REMEH with the Halbach array; (b) REMEH with the two-directional magnet array. 


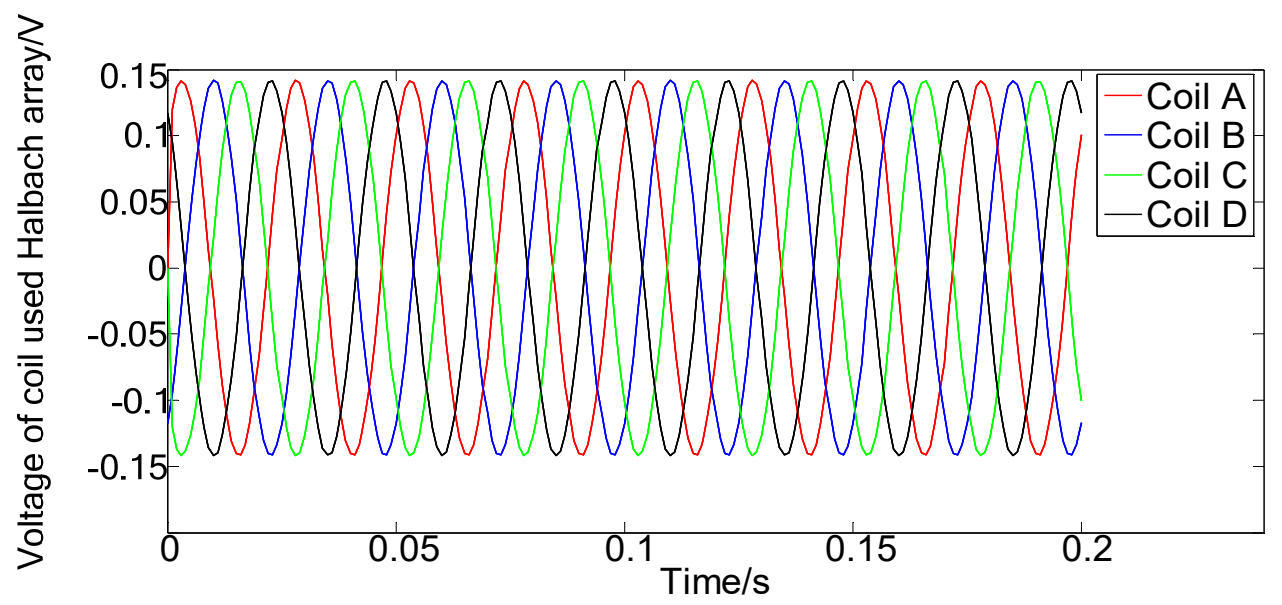

Figure 13. Four phases of the induced voltage in the coil of the REMEH with the Halbach array versus time under a rotation speed of $10 \mathrm{r} / \mathrm{s}$ when the gap diameter is $2 \mathrm{~mm}$.

The induced voltage in the coil of the REMEH using the two-directional magnet array exhibits two different phases, as shown in Figure 14. The induced voltages of the two coils have the same value and opposite directions at the same time. Two induced voltages that are opposite in phase should be connected in series oppositely, thus enhancing the voltage by 2 times [30].

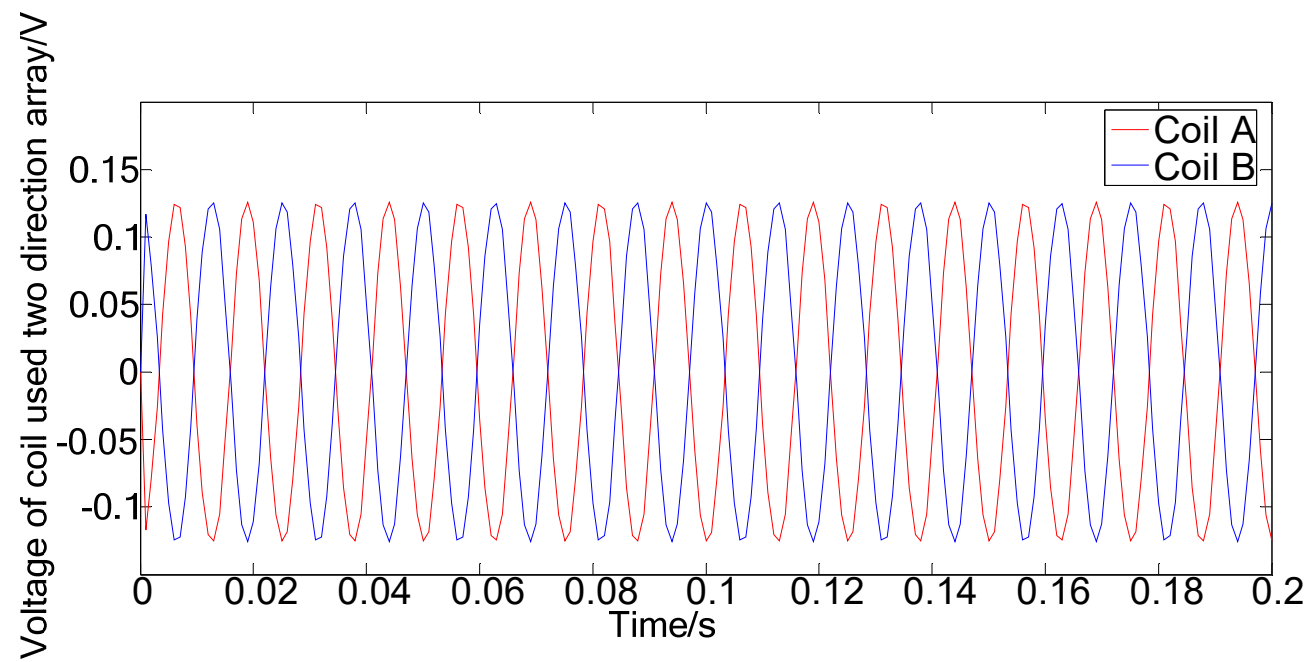

Figure 14. Two phases of the induced voltage in the coil of the REMEH with the two-directional magnet array versus time under a rotation speed of $10 \mathrm{r} / \mathrm{s}$ when the gap diameter is $2 \mathrm{~mm}$.

The RMS value of an induced voltage is an effective way to evaluate the average power of a REMEH. For the proposed REMEH with the Halbach array, as the gap between the magnet and the coil increases, the RMS value of the induced voltage decreases. At the same gap diameter, the RMS value of the induced voltage increases proportionally as the rotation speed increases. For the proposed REMEH with the two-directional magnet array, the RMS value of the induced voltage increases proportionally as the rotation speed increases at the same gap diameter. As the data show in Figure 15, the RMS value of the induced voltage in the REMEH with the two-directional array is obviously larger than that with the Halbach array when the gap diameter is $1 \mathrm{~mm}$. It can be seen from Figures 10-15 that the phase of the induced voltage does not affect the RMS value. The critical gap diameter was determined when the structural parameters were fixed. 


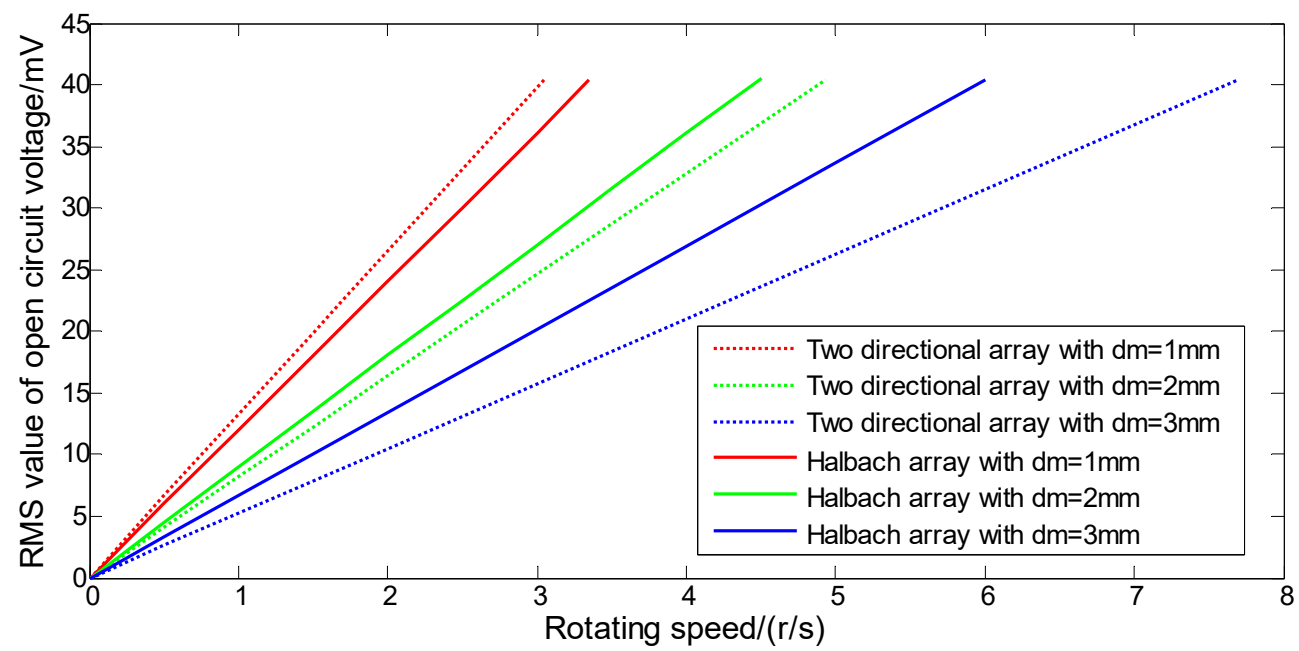

Figure 15. RMS value of the induced voltage of the REMEH with the two-directional magnet array and the REMEH with the Halbach array versus rotation speed at different gap diameters.

As shown in Figure 16, the RMS value of the induced voltage in the coil decreases as the gap between the magnet and the coil increases. The RMS value of the voltage in the coil of the REMEH using the Halbach array decreases more slowly than the RMS value of the voltage in the coil of the REMEH using the two-directional array. When the gap reaches $10 \mathrm{~mm}$, the RMS value of the voltage in the coil of the REMEH with the two-directional magnet array is almost zero while the RMS value of the voltage in the coil of the REMEH with the Halbach array remains considerable. The RMS value of the voltage in the coil of the REMEH formed by the two-directional magnet array or the Halbach array is same when the gap is $1.55 \mathrm{~mm}$. The two-directional array has better performance in energy harvesting than the Halbach array when the gap is less than $1.55 \mathrm{~mm}$. The Halbach array has better performance in energy harvesting than the two-directional array only when the gap is greater than $1.55 \mathrm{~mm}$.

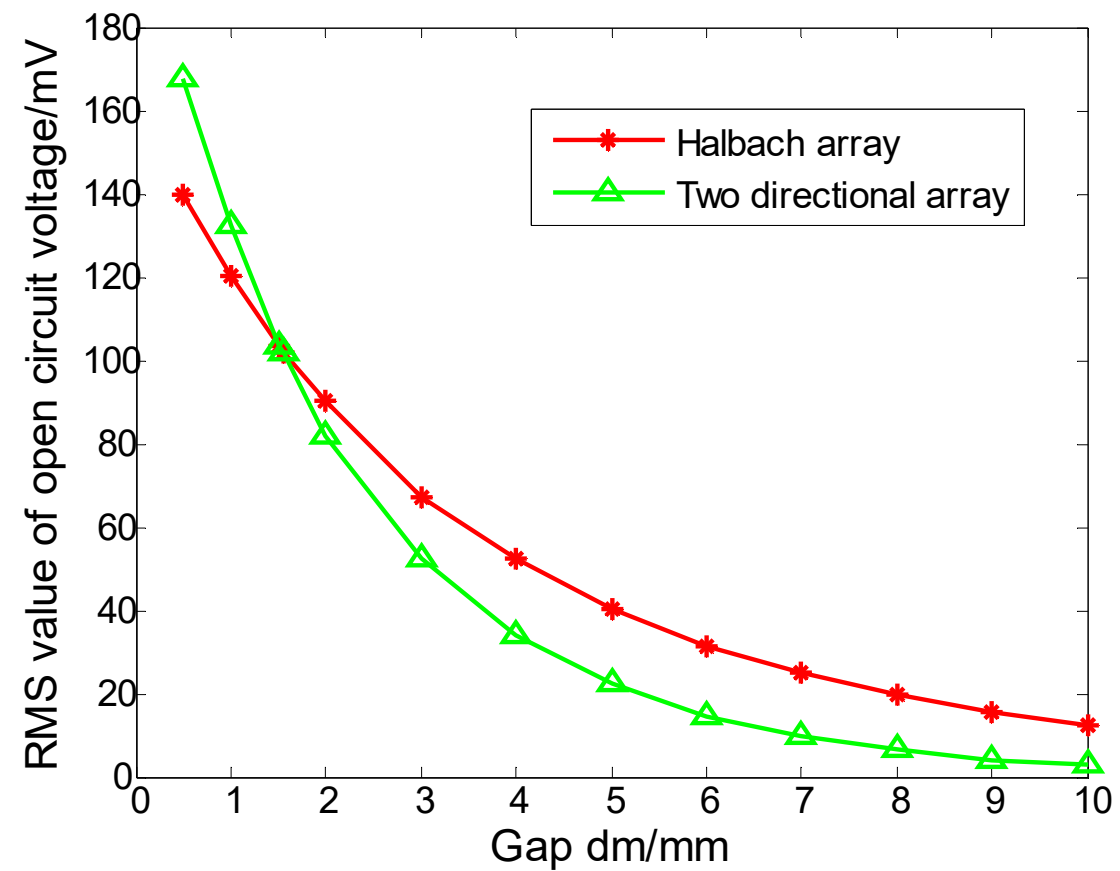

Figure 16. Comparison of the RMS value of the voltage in the REMEH between the Halbach array and the two-directional array under different gaps when the rotation speed is $10 \mathrm{r} / \mathrm{s}$. 


\section{Experimental Verification and Discussion}

\subsection{Prototype Fabrication}

As shown in Figure 17, 16 magnets were embedded in the rotor, which was produced by a 3D printed model in two arrangements (a Halbach array and a two-directional array). The magnet array was connected to the rotating shaft while the coil was fixed on the three-coordinate sliding table. The different rotation speeds of the rotor were provided by the machine. The gap between the coil and the magnet array was controlled by a threecoordinate sliding table using a FZ-740 motor machine manufactured by Feizhi Power Tools Co., Ltd., Ningbo, China. The three-coordinate sliding table was an LD40 produced by PDV corporation. The induced voltage was measured by an oscilloscope (Tektronix MDO3024). The structural parameters of the REMEH are shown in Table 2.

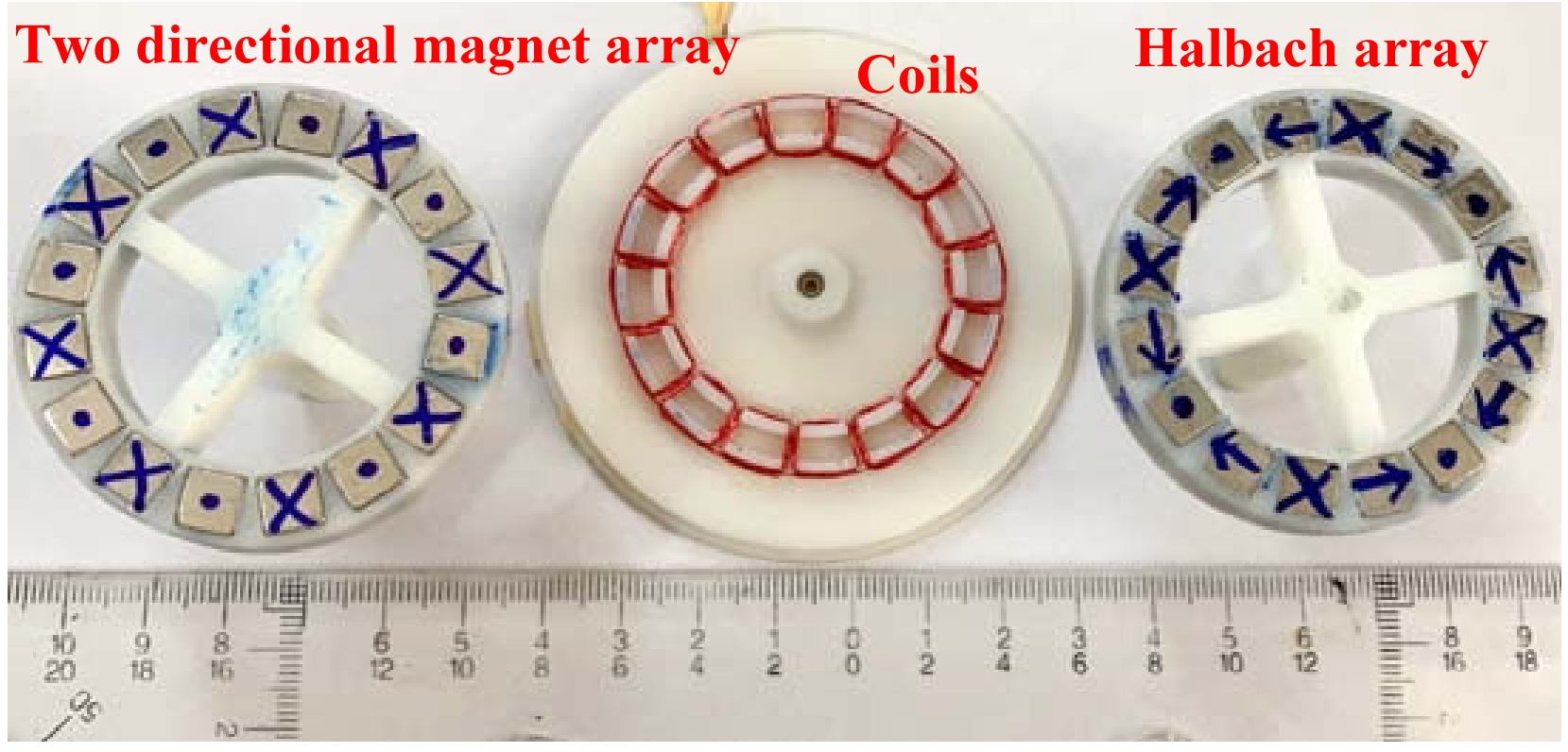

Figure 17. The two kinds of magnet array and coils used in the REMEH (two-directional magnet array, left; Halbach array, right).

Table 2. Material properties and structural parameters.

\begin{tabular}{cc}
\hline Parameters & Value \\
\hline Number of magnets & 16 \\
Material of magnets & $\mathrm{NdFeB}(\mathrm{N35})$ \\
Size of cubic magnets & $7 \mathrm{~mm} \times 7 \mathrm{~mm} \times 7 \mathrm{~mm}$ \\
Norm B & $1.2 \mathrm{~T}$ \\
Turns of each coil & 50 \\
Diameter of wire & $0.1 \mathrm{~mm}$ \\
Inner diameter of magnet ring & $18 \mathrm{~mm}$ \\
Outer diameter of magnet ring & $25 \mathrm{~mm}$ \\
\hline
\end{tabular}

\subsection{Experimental Setup}

The rotation speed between the circular magnet array and the coils was determined by a rotating gain knob. The gap diameter was adjusted by the three-coordinate sliding table. The experimental motor is shown in Figure 18. 


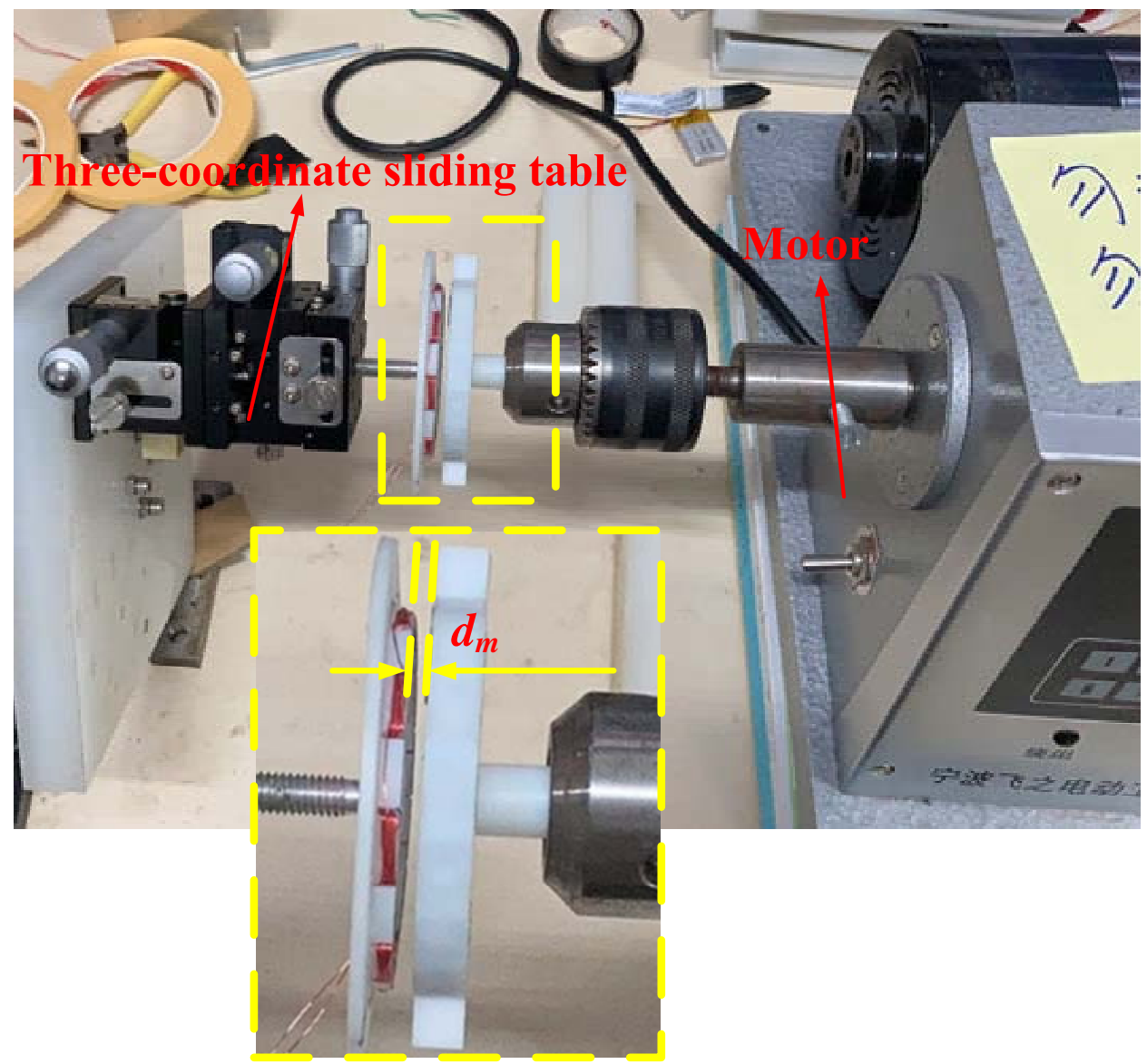

Figure 18. Experiment on the voltage response at certain rotation speeds.

\subsection{Voltage Response at Certain Rotation Speeds}

The simulation voltage response and the experimental voltage response were compared when the rotating speed was fixed. As Figures 19 and 20 show, the measured peak-to-peak values are slightly lower than the simulation results. The frequency of the simulated voltage is consistent with the frequency of the experimental voltage.

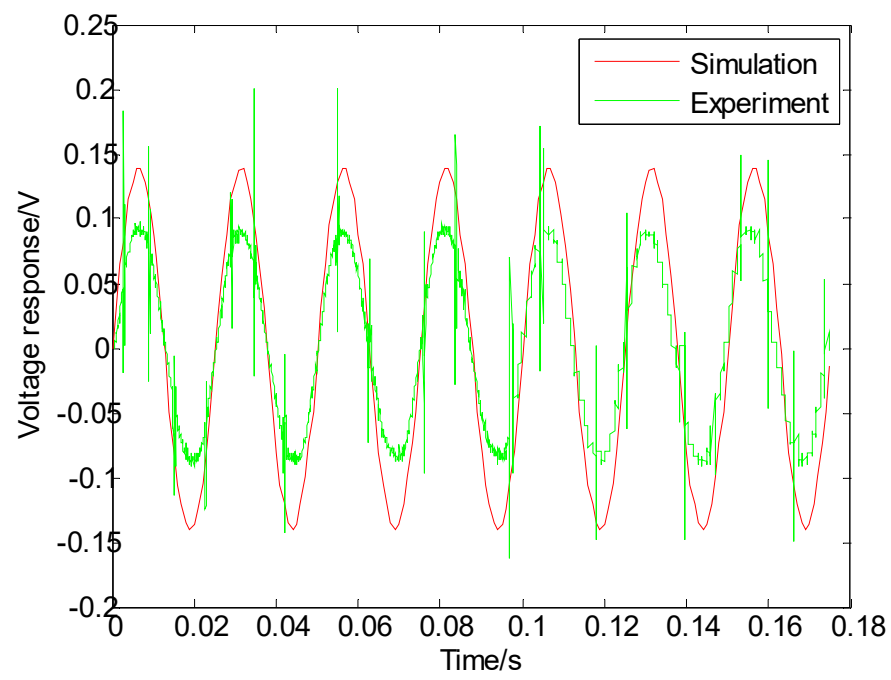

Figure 19. Comparison of the simulated and experimental induced voltage response versus time in the coil when the Halbach array was applied at a gap diameter of $2 \mathrm{~mm}$. 


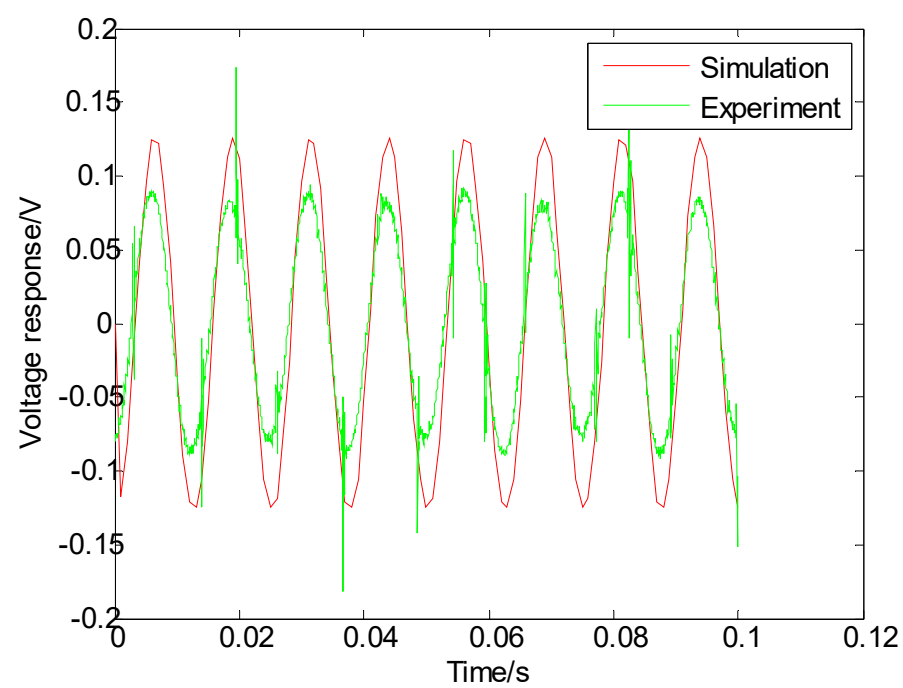

Figure 20. Comparison of the simulated and experimental induced voltage response versus time in the coil when the two-directional array was applied at a gap diameter of $2 \mathrm{~mm}$.

The experimental data and the simulation data were compared at certain rotation speeds and the results are depicted in Figure 21. The RMS value of the induced voltage has a linear relationship with the rotation speed when the distance between the magnet and the coil is $2 \mathrm{~mm}$. The RMS value of the induced voltage in the coil was the same when either of the two magnet arrays was applied in the REMEH.

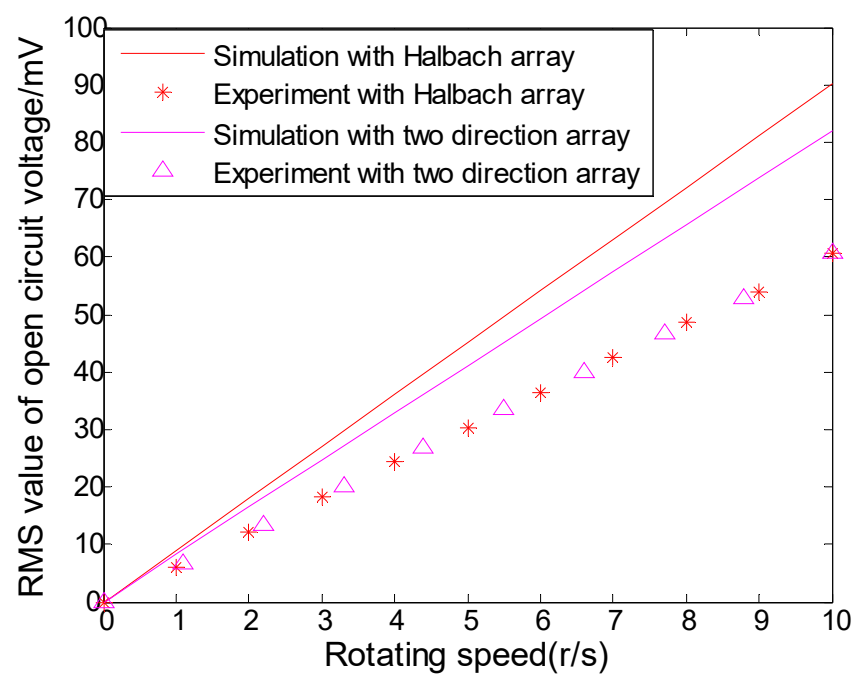

Figure 21. Comparison of the simulation and experimental RMS value of the open-circuit voltage in the coil when the REMEH adopted the two kinds of arrays at a gap of $2 \mathrm{~mm}$.

The RMS value of an induced voltage is an important indicator of energy conversion performance. The average power can be directly compared or calculated according to the RMS value of the induced voltage when the coil parameters are determined. The experimental results in Figure 22 show that the RMS value of the induced voltage in the coil of the REMEH using the two kinds of magnet array decreases as the gap increases at a rotation speed of $10 \mathrm{r} / \mathrm{s}$. The advantages of using Halbach arrays in energy harvesting appear gradually as the gap increases. In the experiments, the RMS value of the voltage in the coils of the REMEH formed by the two-directional magnet array or the Halbach array is the same when the gap is $2 \mathrm{~mm}$. The two-directional array has better performance when the gap is less than $2 \mathrm{~mm}$ and the Halbach array has better performance when the gap is greater than $2 \mathrm{~mm}$. The simulation value of $1.55 \mathrm{~mm}$ and the experimental value 
of $2 \mathrm{~mm}$ exhibit a certain deviation, which is affected by multiple factors. The matching value of the gap diameter, which leads to the same RMS value of the voltage in the coil by the two kinds of magnet array, is determined by $\Delta \mathrm{B}$. The number of magnetic poles of the Halbach array is half that of the two-directional array. Experiments show that although the magnetic flux intensity at the magnetic poles of the Halbach array is higher, the RMS value of the induced voltage in the coil with the two-directional array is larger at a small gap diameter, which is due to the faster change in magnetic flux. The gap between the 16 magnets, the coil, and the cubic magnets in the prototype has a certain deviation that was produced during assembly. In summary, the experimental data are in good agreement with the trend of the simulation data.

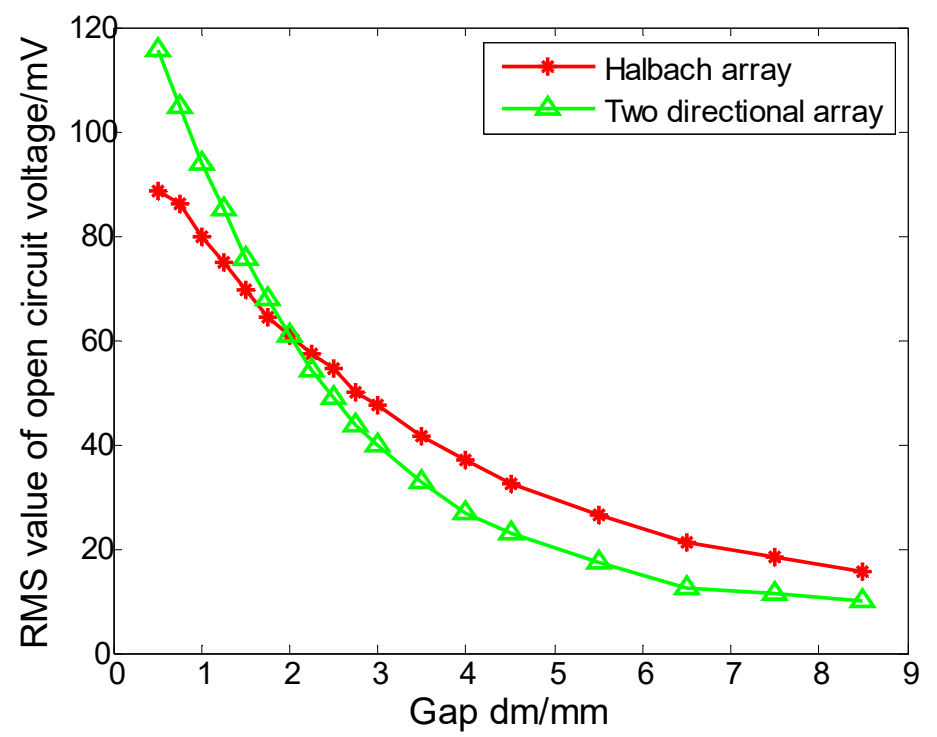

Figure 22. Comparison of the RMS value of the voltage in the REMEH between the Halbach array and the two-directional array under different gaps when the rotation speed was $10 \mathrm{r} / \mathrm{s}$ in the experiment.

\section{Conclusions}

In this study, the application of circular magnet arrays was investigated in terms of energy harvesting performance. Characteristics of the Halbach array with a certain space between the cubic magnets were studied. A theoretical model of an electromagnetic energy harvester in cylindrical coordinates was established based on the coordinates of the cubic magnet nodes. The finite element method was used to calculate the magnetic flux density and induced voltage of the two magnet arrays. The arrangement of the magnets in the Halbach array and the space between the 16 magnets were found to affect the magnetic flux density distribution. In the Halbach array used in this paper, 16 cubic magnets instead of arc magnets were arranged in a special way. The large space between the cubic magnets resulted in the REMEH with the Halbach array having poor energy harvesting performance when the gap was smaller than a threshold value. Through simulation and experimental verification, the induced voltage in the coil under the two kinds of magnet array was found to continuously decrease as the gap between the magnets and the coil increased. The two-directional array has better performance when the gap is less than the threshold gap and the Halbach array has better performance when the gap is greater than the threshold gap. The Halbach array has the characteristic of an enhanced magnetic flux density, which may help with energy harvesting on some special occasions or in a confined space.

In summary, the magnet array should be remanufactured so that there is no space between the magnets when the Halbach array is applied in a REMEH. It can be seen from the simulation results that as the gap diameter increases, the maximum magnetic flux density and the area of the Halbach array's poles become larger than those of the 
two-directional array. The gap between the magnets and the coils and the space between the magnets have obvious effects on the electromagnetic energy conversion.

Author Contributions: S.G. (Shengkai Guo): Conceptualization, Writing-Original Draft; S.G. (Shiqiao Gao): Methodology, Supervision; L.J.: Investigation, Formal Analysis; X.D.: Data Curation, Software; Q.W.: Editing, Visualization; J.S.: Supervision, Visualization. All authors have read and agreed to the published version of the manuscript.

Funding: This research received no external funding.

Institutional Review Board Statement: Not applicable.

Informed Consent Statement: Not applicable.

Data Availability Statement: Not applicable.

Conflicts of Interest: The authors declare no conflict of interest.

\section{References}

1. Liu, H.; Hou, C.; Lin, J.; Li, Y.; Shi, Q.; Chen, T.; Sun, L.; Lee, C. A non-resonant rotational electromagnetic energy harvester for low-frequency and irregular human motion. Appl. Phys. Lett. 2018, 113, 203901. [CrossRef]

2. Zhou, N.; Zhang, Y.; Bowen, C.R.; Cao, J. A stacked electromagnetic energy harvester with frequency up-conversion for swing motion. Appl. Phys. Lett. 2020, 117, 163904. [CrossRef]

3. Zhao, L.C.; Zou, H.X.; Gao, Q.H.; Yan, G.; Liu, F.R.; Tan, T.; Wei, K.X.; Zhang, W.M. Magnetically modulated orbit for human motion energy harvesting. Appl. Phys. Lett. 2019, 115, 263902. [CrossRef]

4. Zhang, Y.; Luo, A.; Wang, Y.; Dai, X.; Lu, Y.; Wang, F. Rotational electromagnetic energy harvester for human motion application at low frequency. Appl. Phys. Lett. 2020, 116, 053902.

5. Cai, M.; Wang, J.; Liao, W.-H. Self-powered smart watch and wristband enabled by embedded generator. Appl. Energy 2020, 263, 114682. [CrossRef]

6. Salauddin, M.; Rasel, M.S.; Kim, J.W.; Park, J.Y. Design and experiment of hybridized electromagnetic-triboelectric energy harvester using Halbach magnet array from handshaking vibration. Energy Convers. Manag. 2017, 153, 1-11. [CrossRef]

7. Salauddin, M.; Toyabur, R.M.; Maharjan, P.; Park, J.Y. High performance human-induced vibration driven hybrid energy harvester for powering portable electronics. Nano Energy 2018, 45, 236-246. [CrossRef]

8. Salauddin, M.; Park, J.Y. A low frequency vibration driven, miniaturized and hybridized electromagnetic and triboelectric energy harvester using dual Halbach array. In Proceedings of the 2017 19th International Conference on Solid-State Sensors, Actuators and Microsystems (TRANSDUCERS), Kaohsiung, Taiwan, 18-22 June 2017.

9. Salauddin, M.; Toyabur, R.M.; Maharjan, P.; Rasel, M.S.; Kim, J.W.; Cho, H.; Park, J.Y. Miniaturized springless hybrid nanogenerator for powering portable and wearable electronic devices from human-body-induced vibration. Nano Energy 2018, 51, 61-72. [CrossRef]

10. Rana, S.S.; Rahman, M.T.; Salauddin, M.; Maharjan, P.; Bhatta, T.; Cho, H.; Park, J.Y. A human-machine interactive hybridized biomechanical nanogenerator as a self-sustainable power source for multifunctional smart electronics applications. Nano Energy 2020, 76, 105025. [CrossRef]

11. Ma, C.; Zhao, W.; Qu, L. Design optimization of a linear generator with dual Halbach array for human motion energy harvesting In Proceedings of the 2015 IEEE International Electric Machines \& Drives Conference (IEMDC), Coeur d'Alene, ID, USA, 10-13 May 2015; pp. 703-708.

12. Sajwani, H.; Al Suwaidi, N.; Elshikh, M.; Al Jaberi, A.; Meribout, M. Hallbach array-based linear generator for human motion energy harvesting. In Proceedings of the 2017 International Conference on Electrical and Computing Technologies and Applications (ICECTA), Ras Al Khaimah, United Arab Emirates, 21-23 November 2017; pp. 1-6.

13. Li, Z.; Yan, Z.; Luo, J.; Yang, Z. Performance comparison of electromagnetic energy harvesters based on magnet arrays of alternating polarity and configuration. Energy Convers. Manag. 2019, 179, 132-140. [CrossRef]

14. Wang, Y.; Zhang, Q.; Zhao, L.; Tang, Y.; Shkel, A.; Kim, E.S. Vibration energy harvester with low resonant frequency based on flexible coil and liquid spring. Appl. Phys. Lett. 2016, 109, 203901. [CrossRef]

15. Liu, N.; Tan, Y.; Mo, W.; Han, H.; Li, L. Optimization Design for Ocean Wave Energy Convertor. In Proceedings of the 2020 International Conference on Energy, Environment and Bioengineering (ICEEB 2020), Xi'an, China, 7-9 August 2020; Volume 185, p. 01073. [CrossRef]

16. Nico, V.; Boco, E.; Frizzell, R.; Punch, J. A high figure of merit vibrational energy harvester for low frequency applications. Appl. Phys. Lett. 2016, 108, 013902. [CrossRef]

17. Tan, Y.; Liu, N.; Lin, K.; Zhang, Z. Frequency Domain Modeling of a Halbach PM Linear Generator Based Two-Body Point Absorber for Wave Energy Conversion. Front. Energy Res. 2020, 8, 19. [CrossRef] 
18. Shahosseini, I.; Najafi, K. Cylindrical halbach magnet array for electromagnetic vibration energy harvesters. In Proceedings of the 2015 28th IEEE International Conference on Micro Electro Mechanical Systems (MEMS), Estoril, Portugal, 18-22 January 2015; pp. 1051-1054.

19. Liu, X.; Qiu, J.; Chen, H.; Xu, X.; Wen, Y.; Li, P. Design and Optimization of an Electromagnetic Vibration Energy Harvester Using Dual Halbach Arrays. IEEE Trans. Magn. 2015, 51, 8204204. [CrossRef]

20. Qiu, J.; Liu, X.; Chen, H.; Xu, X.; Wen, Y.; Li, P. A Low-Frequency Resonant Electromagnetic Vibration Energy Harvester Employing the Halbach Arrays for Intelligent Wireless Sensor Networks. IEEE Trans. Magn. 2015, 51, 8600604. [CrossRef]

21. Qiu, J.; Liu, X.; Hu, Z.; Chang, Q.; Gao, Y.; Yang, J.; Wen, J.; Tang, X.; Hu, W. Multi-directional electromagnetic vibration energy harvester using circular Halbach array. AIP Adv. 2017, 7, 056672. [CrossRef]

22. Zhu, D.; Beeby, S.; Tudor, J.; Harris, N. Vibration energy harvesting using the Halbach array. Smart Mater. Struct. 2012, 21, 075020. [CrossRef]

23. Zhu, D.; Beeby, S.; Tudor, J.; Harris, N. Increasing output power of electromagnetic vibration energy harvesters using improved Halbach arrays. Sens. Actuat A Phys. 2013, 203, 11-19. [CrossRef]

24. Kim, J.W.; Salauddin, M.; Cho, H.; Rasel, M.S.; Park, J.Y. Electromagnetic energy harvester based on a finger trigger rotational gear module and an array of disc Halbach magnets. Appl. Energy 2019, 250, 776-785. [CrossRef]

25. Wang, Y.-J.; Chen, C.-D.; Sung, C.-K.; Li, C. Natural frequency self-tuning energy harvester using a circular Halbach array magnetic disk. J. Intel. Mat. Syst. Str. 2012, 23, 933-943. [CrossRef]

26. Zhang, Y.; Cao, J.; Zhu, H.; Lei, Y. Design, modeling and experimental verification of circular Halbach electromagnetic energy harvesting from bearing motion. Energy Convers. Manag. 2019, 180, 811-821. [CrossRef]

27. Zhang, Y.; Cao, J.; Liao, W.-H.; Zhao, L.; Lin, J. Theoretical modeling and experimental verification of circular Halbach electromagnetic energy harvesters for performance enhancement. Smart Mater. Struct. 2018, 27, 095019. [CrossRef]

28. Maamer, B.; Kaziz, S.; Boughamoura, A.; Jaziri, N.; Tounsi, F. Numerical Modeling of a Novel Mesoscale EM Energy Harvesting System Based on Halbach Array. In Proceedings of the 17th IEEE International Multi-Conference on Systems, Signals \& Devices, Monastir, Tunisia, 20-23 July 2020; pp. 495-500.

29. Bancel, F. Magnetic nodes. J. Phys. D Appl. Phys. 1999, 32, 2155-2161. [CrossRef]

30. Guo, S.; Gao, S.; Jin, L.; Du, X.; Yin, Z.; Wu, Q.; Li, Z. Mechanism, theory and application research of a rotating electromagnetic energy harvester suitable for multi-directional excitation. J. Phys. D Appl. Phys. 2022, 55, 085503. [CrossRef] 